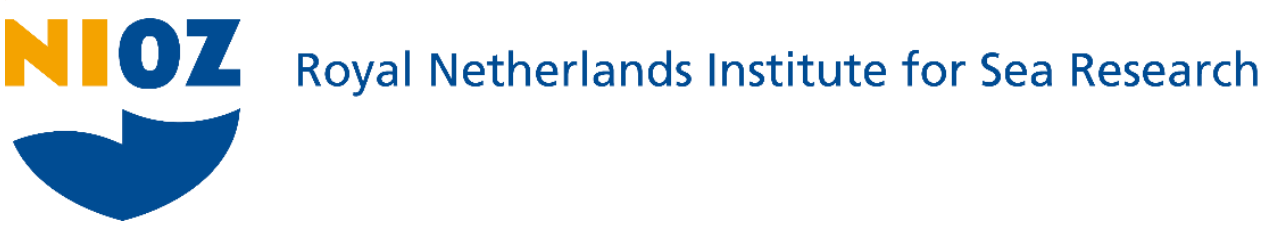

This is a pre-copyedited, author-produced version of an article accepted for publication, following peer review.

Cornacchia, L.; Licci, S.; Nepf, H.; Folkard, H.; van der Wal, D.; van de Koppel, J.; Puijalon, S. \& Bouma, T.J. (2019). Turbulence-mediated facilitation of resource uptake in patchy stream macrophytes. Limnology and Oceanography, 64, 714-727

Published version: https://dx.doi.org/10.1002//no.11070

Link NIOZ Repository: http://www.vliz.be/nl/imis?module=ref\&refid=303426

[Article begins on next page]

The NIOZ Repository gives free access to the digital collection of the work of the Royal Netherlands Institute for Sea Research. This archive is managed according to the principles of the Open Access Movement, and the Open Archive Initiative. Each publication should be cited to its original source - please use the reference as presented.

When using parts of, or whole publications in your own work, permission from the author(s) or copyright holder(s) is always needed. 


\section{Turbulence-mediated facilitation of resource uptake in patchy stream macrophytes}

2 Authors:

3 Loreta Cornacchia ${ }^{1,5, \text { a }}$

4 Sofia Licci $^{2}$

$5 \quad$ Heidi Nepf ${ }^{3}$

6 Andrew Folkard ${ }^{4}$

7 Daphne van der $\mathrm{Wal}^{1,6}$

8 Johan van de Koppel ${ }^{1,5}$

9 Sara Puijalon ${ }^{2}$

10 Tjeerd J. Bouma ${ }^{1,5}$

\author{
loreta.cornacchia@nioz.nl, loreta.cornacchia@univ-lyon1.fr \\ sofia.licci@univ-lyon1.fr \\ hmnepf@mit.edu \\ a.folkard@lancaster.ac.uk \\ daphne.van.der.wal@nioz.nl \\ johan.van.de.koppel@nioz.nl \\ sara.puijalon@univ-lyon1.fr \\ tjeerd.bouma@nioz.nl
}

\section{Affiliations:}

${ }^{1}$ NIOZ Royal Netherlands Institute for Sea Research, Department of Estuarine and Delta Systems, and Utrecht University, P.O. Box 140, 4400 AC Yerseke, the Netherlands.

${ }^{2}$ Univ Lyon, Université Claude Bernard Lyon 1, CNRS, ENTPE, UMR 5023 LEHNA, F-69622, Villeurbanne, France

${ }^{3}$ Dept. of Civil and Environmental Engineering, Massachusetts Institute of Technology, 77 Massachusetts Ave., Bldg. 48-216D, Cambridge, MA 02139, USA.

${ }^{4}$ Lancaster Environment Centre, Lancaster University, Lancaster, UK

${ }^{5}$ Groningen Institute for Evolutionary Life Sciences, University of Groningen, PO Box 11103, 9700 CC Groningen, The Netherlands

${ }^{6}$ Faculty of Geo-Information Science and Earth Observation (ITC), University of Twente, P.O. Box 217, 7500 AE, Enschede, The Netherlands

a Present address: UMR 5023 LEHNA, Université Lyon 1, CNRS, ENTPE, Villeurbanne Cedex, France

Corresponding author:

Loreta Cornacchia

Université Lyon 1

CNRS, UMR 5023 - LEHNA

Laboratoire d'Ecologie des Hydrosystèmes Naturels et Anthropisés

6, rue Raphaël Dubois - Bât. Forel

F-69622 Villeurbanne Cedex FRANCE

e.mail: loreta.cornacchia@univ-lyon1.fr

Tel.: $\quad+33(0) 472431293$

Fax: $\quad+33(0) 472431141$ 
39 Running head: Turbulence-driven facilitation in macrophytes

$40 \quad$ Keywords: submerged macrophytes; nutrient uptake; mass transfer 


\section{Abstract}

42 Many landscapes are characterized by a patchy, rather than homogeneous, distribution of vegetation.

43 Often this patchiness is composed of single-species patches with contrasting traits, interacting with

44 each other. To date, it is unknown whether patches of different species affect each other's uptake of

45

46

47 resources by altering hydrodynamic conditions, and how this depends on their spatial patch configuration. Patches of two contrasting aquatic macrophyte species (i.e., dense canopy-forming Callitriche and sparse canopy-forming Groenlandia) were grown together in a racetrack flume and placed in different patch configurations. We measured ${ }^{15} \mathrm{NH}_{4}{ }^{+}$uptake rates and hydrodynamic properties along the centerline and the lateral edge of both patches. When the species with a taller, denser canopy (Callitriche) was located upstream of the shorter, sparser species (Groenlandia), it generated turbulence in its wake that enhanced nutrient uptake for the sparser Groenlandia. At the same time, Callitriche benefited from being located at a leading edge where it was exposed to higher mean velocity, as its canopy was too dense for turbulence to penetrate from upstream. Consistent with this, we found that ammonium uptake rates depended on turbulence level for the sparse Groenlandia and on mean flow velocity for the dense Callitriche, but Total Kinetic Energy was the best descriptor of uptake rates for both species. By influencing turbulence, macrophyte species interact with each other through facilitation of resource uptake. Hence, heterogeneity due to multi-specific spatial patchiness has crucial implications for both species interactions and aquatic ecosystem functions, such as nitrogen retention. 


\section{Introduction}

61 In many ecosystems, vegetation shapes entire landscapes by interacting with physical processes

62

63

64

65

66

67

(Dietrich and Perron 2006; Corenblit et al. 2011). In coastal and fluvial aquatic ecosystems, vegetation modifies habitats through its effects on hydrodynamics and sedimentation (Leonard and Luther 1995; Madsen et al. 2001; Schulz et al. 2003; Bouma et al. 2007), hence acting as an ecosystem engineer (Jones et al. 1994). Many studies first considered interactions between hydrodynamics and homogeneous vegetation (Kouwen and Unny 1973; Nepf 1999; Nepf and Vivoni 2000; Järvelä 2005; Chen et al. 2013), and later focused on isolated or pairs of patches (Sand-Jensen and Vindbœk Madsen 1992; Folkard 2005; Bouma et al. 2009; Vandenbruwaene et al. 2011; Chen et al. 2012; Zong and Nepf 2012). Generally, vegetation patches locally reduce flow velocities, while increasing them in some adjacent areas (Bouma et al. 2007; Chen et al. 2012; Schoelynck et al. 2012; Meire et al. 2014).

In aquatic ecosystems, the interaction between vegetation and hydrodynamics regulates important ecological processes such as nutrient delivery and uptake by plants, as nutrients can be taken up from the water column through plant shoots (Madsen and Cedergreen 2002; Bal et al. 2013). These processes are crucial for community primary productivity and ecosystem function (Thomas et al. 2000; Morris et al. 2008; Levi et al. 2015). Previous studies on uptake rates in relation to hydrodynamic conditions mainly focused on seagrasses, using flume experiments with dissolved ${ }^{15} \mathrm{~N}$-labeled ammonium or nitrate (often the main inorganic nitrogen sources in natural conditions; Haynes and Goh (1978)). These works identified the important effects of water velocity and flow alteration by seagrass canopies on resource uptake (Thomas et al. 2000; Cornelisen and Thomas 2006), and the dependence of uptake rates on the rate of mass transfer to the leaf surface under unidirectional flow (Cornelisen and Thomas 2004). Further, Morris et al. (2008) identified spatial patterns in ammonium uptake within seagrass patches, with higher uptake observed at the leading edge of the patch where the turbulent kinetic energy and velocity within the patch were highest. In a study of nutrient uptake by river 
macrophytes, Bal et al. (2013) found that ammonium uptake increased with flow velocity. Because the diffusive boundary layer decreases with increasing velocity, the uptake rate also increases with increasing velocity (Thomas et al. 2000; Cornelisen and Thomas 2004; Morris et al. 2008; Bal et al. 2013).

However, most of the previous studies dealt with monospecific canopies or focused on a single species at a time, creating a monospecific community, while in reality natural landscapes are a diverse community made up of multiple species. Different patches of single species are heterogeneously distributed, and this patchiness is a common characteristic of aquatic habitats (Sand-Jensen and Vindbœk Madsen 1992). A few examples are patchy seagrass meadows (Fonseca et al. 1983), and streams characterized by a 'pseudo-braided' distribution of plant stands between areas of faster flow (Dawson and Robinson 1984; Cotton et al. 2006; Wharton et al. 2006). This additional level of complexity has just started to be integrated in studies of hydrodynamic-vegetation interactions. For instance, Weitzman et al. (2015) focused on hydrodynamic implications of multi-specific canopies, but considered canopy heterogeneity in the vertical dimension. Adhitya et al. (2014) focused on hydrodynamics and spatial configurations of seagrass patches with different densities, but did not test the consequences for resource uptake. Bal et al. (2013) focused on nutrient uptake rates within monospecific patches of two species next to each other, but they only tested a single spatial configuration and therefore did not investigate the effects of spatial patchiness. To date, it is still unknown how patches of different species interact with each other by altering hydrodynamics and uptake of resources, and how this depends on their landscape configuration.

Multispecies effects could be important for hydrodynamics and nutrient uptake because the density, flexibility and canopy structure of different species affect hydrodynamics differently (Peralta et al. 2008; Bouma et al. 2013). As we cannot easily predict the flow alteration by heterogeneous species distributions, our understanding of the implications for species interactions and nutrient load 
reduction in aquatic ecosystems is limited. Generally, the hydrodynamic controls on uptake rate are expected to be dependent on the macro-scale rate of delivery (mean flow velocity; e.g. Cornelisen and Thomas (2006)), or on the micro-scale processes that determine the concentration gradient at the leaf

111 boundary layer (turbulence; e.g. Morris et al. (2008)). However, in a diverse community there might be cases where a single hydrodynamic parameter is not sufficient to describe uptake rates for multiple species with different traits and effects on hydrodynamic conditions. For instance, turbulence can have a significantly smaller scale in very dense canopies, compared to sparser ones (Nepf 2012). On the other hand, the mean flow speed can be relatively constant within sparse canopies, but turbulence might be locally variable. Therefore, understanding the interaction between multiple species in terms of nutrient uptake, mediated by their hydrodynamic effects, is essential to gain a more realistic understanding of species interactions and productivity in heterogeneous, multi-specific communities. In this study, we use streams colonized by aquatic macrophytes as a model system. We investigate how patches of two different species with contrasting morphological traits interact with each other by influencing hydrodynamics, and thereby ammonium uptake. Moreover, we test how this depends on their spatial configuration (patchiness). Here, we define multi-specific patchiness as a community composed of patches of different species. Specifically, we study the interaction between two macrophyte species that co-occur under field conditions and have contrasting density and canopy structure. Callitriche platycarpa Kütz., 1842 forms very dense patches that exhibit increasing canopy height with increasing patch length ('dense' species). Groenlandia densa (L.) Fourr. has a more open canopy, and its canopy height is constant along the patch length ('sparse' species). In the field, the dense patches of Callitriche are distributed quite regularly at a distance of about 8 meters, and Groenlandia patches tend to aggregate around them (Cornacchia et al. 2018). Given the differences in

130 shoot density and canopy architecture between the two species (Table 1), we hypothesize that the 131 effects of the dense Callitriche patches on hydrodynamics may facilitate the delivery and uptake of 
132 resources by the sparse Groenlandia patches. To test this hypothesis, patches of the two species were

133 arranged in different configurations in a laboratory flume. To investigate the role of spatial

134 configuration and reciprocal species effects on nutrient uptake, both the species upstream and the

135 relative location of the species downstream were varied. We discuss the implications of multi-specific

136 spatial patchiness on facilitation and aquatic ecosystem functions, such as nitrogen retention.

\section{Materials and methods}

\section{$138 \quad$ Plant material}

139 We tested the effect of macrophyte patch species and configuration on ammonium uptake rates using

140 two submerged macrophytes species, Callitriche platycarpa and Groenlandia densa. Both species were

141 collected in February 2015 from a wetland on the Ain River, France (5.2825 ${ }^{\circ}$, 45.9855 N). Plants

142 were stored in plastic bags and transported to the laboratory in NIOZ Yerseke (The Netherlands) within

14324 hours from collection. Until installation in the flume, the two macrophyte species were stored in a

144 green house, in tanks filled with freshwater that was continuously aerated, and exposed to natural light.

145 The macrophytes were allowed to recover for two days in the green house before starting the

146 experiments. In order to be used for the experimental setup, individual plants were transplanted in

147 stainless steel trays $(30 \times 29.5 \times 5 \mathrm{~cm})$. The trays were filled with a bottom layer of river sand $(4.5 \mathrm{~cm})$

148 and a top layer $(0.5 \mathrm{~cm})$ of fine gravel $(0.2 \mathrm{~cm}$ grain size $)$. A false bottom in the flume allowed the trays

149 to be inserted with the soil surface at the same level as the flume bed. Based on the naturally occurring

150 densities of the two species in the field, we constructed patches of $97 \pm 28 \mathrm{~g} \mathrm{DW} \mathrm{m}^{-2}$ (mean \pm SD) for

151 Groenlandia ('sparse' species) and $318 \pm 67 \mathrm{~g} \mathrm{DW} \mathrm{m}^{-2}$ for Callitriche ('dense' species) (Figure 1;

152 Table 1). The biomass of the constructed patches was estimated by collecting all plant material within

153 randomly selected $0.1 \times 0.1 \mathrm{~m}$ quadrants $(n=3)$. The mean patch biomass for Callitriche is close to the 
154 range of $208-256 \mathrm{~g} \mathrm{DW} \mathrm{m}^{-2}$ reported in Sand-Jensen and Vindbœk Madsen (1992). The mean patch

155 biomass for Groenlandia corresponded to ca. 500 shoots $\mathrm{m}^{-2}$, which represents a relatively low shoot

156 density in natural plant beds (Sheldon and Boylen 1977). We used a different patch length for each

157 species to resemble the typical lengths observed in the field, i.e. $2.7 \mathrm{~m}$ and $1.2 \mathrm{~m}$ on average for

158 Groenlandia and Callitriche respectively. These values are representative of average patch sizes

159 observed in field conditions $(2.5 \pm 1.7 \mathrm{~m}$ for Groenlandia, $n=20 ; 1.4 \pm 0.8 \mathrm{~m}$ for Callitriche, $n=20$;

160 Supporting Information Fig. S1). We used a total of 9 trays for Groenlandia, for a total patch coverage

161 of $2.7 \times 0.3 \mathrm{~m}$. For Callitriche, plants were rooted in two trays $(0.6 \times 0.3 \times 0.05 \mathrm{~m})$. When Callitriche

162 was placed upstream, three trays (filled with the same soil as the plant trays) were placed between the

163 two patches, to account for the presence of the typical overhanging canopy for this species. That is,

164 when the flume was running, a total coverage of $1.20 \times 0.3 \mathrm{~m}^{2}$ was observed due to shoots bending;

165 this region was considered as part of the Callitriche patch (see Figure 2). A distance of one tray (0.3 m)

166 between the two patches was used for the configurations in which Groenlandia was in the upstream

167 position. The flume section next to each patch was left open (without plants, but filled with the same

168 soil substrate used in the plant trays) in all configurations. Thus, the patches occupied one half of the

169 flume, rather than extending across the width of the flume. As patches in the field do not span the

170 whole channel, this configuration is representative of the typical distribution of vegetation patches in

171 streams, with an empty (unvegetated) zone next to the patch into which water flow is deflected and

172 accelerated around the patch (Fonseca et al. 1983; Gambi et al. 1990; Bouma et al. 2007; Follett and

173 Nepf 2012). The canopies of both species were fully submerged during the experiments. The relative

174 depth of submergence $(\mathrm{H} / \mathrm{h}$, ratio of water depth to maximum canopy height; Nepf (2012)) was

175 relatively constant along the Groenlandia patch due to its uniform height. Values of $\mathrm{H} / \mathrm{h}$ ranged

176 between 8.75 and 4.3 , corresponding to shallow submerged $(\mathrm{H} / \mathrm{h}<5)$. Instead, canopy height along the

177 Callitriche patch varied from $\mathrm{H} / \mathrm{h}=17.5$ at the leading edge (i.e. deeply submerged, $\mathrm{H} / \mathrm{h}>10$ ) to 3.0 in 
178 the middle of the patch (indicating shallow submergence $(\mathrm{H} / \mathrm{h}<5)$, and even 1.30 at the downstream

179 end of the patch, which is very close to emergent conditions $(\mathrm{H} / \mathrm{h}=1)$.

\section{Flume setup and experimental configurations}

181 All experiments were performed within a unidirectional racetrack flume using a water depth of $0.35 \mathrm{~m}$ and with a cross-sectionally-averaged velocity of $0.24 \pm 0.03 \mathrm{~m} \mathrm{~s}^{-1}$. This is a moderate flow velocity, representative of the summer flow conditions in streams typically colonized by Callitriche and Groenlandia $\left(0.21 \pm 0.01 \mathrm{~m} \mathrm{~s}^{-1}\right.$, based on our field measurements in 2014 and 2015; Cornacchia et al. (2018)). For a more detailed description of the flume, see Bouma et al. (2005). To test for the effects of patch spatial configuration on ammonium uptake rates, the two patches were arranged one downstream of the other, either on the same side of the flume ('aligned' configurations), or on opposite sides ('staggered' configurations) (Figure 2). These different spatial configurations are both commonly observed in natural streams, where patches grow downstream of other patches, or in a staggered arrangement (L. Cornacchia, pers. obs.). Moreover, patches of different species in the field can be found co-occurring at very short distances from each other, at the scale of $0.5 \mathrm{~m}$ (Cornacchia et al. 2018). To test for interactions between the two species, in terms of reciprocal effects on ammonium uptake rates, we also switched the species located upstream for each of these configurations ('SparseDense' or 'Dense-Sparse' configurations).

\section{Measuring spatial patterns in ${ }^{15} \mathrm{~N}-\mathrm{NH}_{4}{ }^{+}$uptake rates and canopy hydrodynamics}

To determine spatial patterns of ammonium uptake rates by the macrophyte species, we measured uptake rates at selected locations within the patches (Figure 2). Nutrient uptake rates were determined inside the two patches at $10 \%, 50 \%$ and $90 \%$ of the patch length $(0.27,1.45$ and $2.43 \mathrm{~m}$ from the leading edge in Groenlandia; 0.12, 0.6 and $1 \mathrm{~m}$ from the leading edge in Callitriche) and, for each location along the patch length, at 0.15 and $0.25 \mathrm{~m}$ of the patch width. For each incubation experiment, 
macrophyte individuals were randomly selected from the tanks where they were kept with freshwater and were transplanted into plastic pots ( 5 shoots per pot). Before transplantation in the flume, roots were removed from the plants at the selected test locations, to prevent ammonium uptake by that means from the labeled water that inevitably penetrated into the sand (following Bal et al. (2013)). Since the sediment was not changed in between treatments (as that would have meant destroying and recreating the patches), this avoided an effect of treatment order on uptake rates. While some nutrients are obtained through roots in field conditions, the nutrient demands of many macrophyte species can be satisfied by shoot nutrient uptake alone (Madsen and Cedergreen 2002). Although root removal may have potentially affected the plant response to the flow, it was unlikely to affect their ability to resist the flow during a short-term experiment (6 hours). Each plastic pot was then placed in one of the patch locations described above, and inserted in the trays so that their upper part was in line with the sediment level to avoid scouring effects. The pots were replaced after each incubation experiment and new plants were transplanted. The renewal of plants between each run allowed us to avoid an effect of treatment order on uptake rates, due to plants being exposed to the labeled water for a longer time. Moreover, it provided natural variability in plant structure, while maintaining a constant patch structure by keeping the trays forming the plant patches in the same positions between runs. The treatments were 217 run in the order shown in Figure 1.

In the incubation experiments, ${ }^{15} \mathrm{~N}_{-} \mathrm{NH}_{4}{ }^{+}$was added to the water creating a 20 to $30 \mu \mathrm{mol}-$ $\mathrm{NH}_{4}{ }^{+} \mathrm{L}^{-1}$ solution, with $30 \%$ of the $\mathrm{N}$ as ${ }^{15} \mathrm{~N}$ abundance, following Bal et al. (2013). This range of values is representative of nutrient concentrations found in natural ecosystems: in summer 2015, average ammonium levels recorded in the studied region were $24.5 \pm 28.54 \mu$ mol- $\mathrm{NH}_{4}{ }^{+} \mathrm{L}^{-1}$, with a maximum value of $79.6 \mu$ mol- $\mathrm{NH}_{4}{ }^{+} \mathrm{L}^{-1}$. Such a high enrichment $(30 \%)$ was chosen to minimize dilution effects of the ${ }^{15} \mathrm{~N}$ source pool over the course of the experiment. At the start and end of the experiment, three replicate water samples were taken to measure $\mathrm{NH}_{4}{ }^{+}$concentration in the water. The 
same labeled water was used to perform four experiments, before replacing it with freshwater and a new label for the next runs (based on Bal et al. (2013)). Incubations were performed under artificial light conditions. Lamps were mounted above the flume tank throughout the test section to provide $14 \mathrm{~h}$ $\mathrm{d}^{-1}$ of light (photosynthetic photon flux density of $550 \mu \mathrm{mol} \mathrm{m} \mathrm{m}^{-2} \mathrm{~s}^{-1}$; measured $35 \mathrm{~cm}$ above the sediment surface). The stable isotope was added near the paddles that drive the flow in the flume to ensure mixing. Each incubation experiment lasted for 6 hours, and two replicate runs were performed for each configuration. At the end of the 6 hours, macrophytes were collected from the test positions, rinsed with tap water to remove excess isotope from the plant surface, and folded into aluminum foil. In addition to the samples collected ( $n=30$ for each species), five specimens per species were randomly selected during the experiments from our species stock, to determine the background ${ }^{15} \mathrm{~N}$ signal. The plants were dried in the oven for 48 hours at $60^{\circ} \mathrm{C}$, and individual biomass was weighed. Dried macrophytes were ground to a fine powder using a ball mill (MM 2000, Retsch, Haan,

Germany). A subsample of about $3 \mathrm{mg}$ of powder per plant was sent to the laboratory for mass spectrometry analysis of the isotope ratio. The samples were analysed for total $\mathrm{N}$ content and ${ }^{15} \mathrm{~N}$ atomic percentage $\left(\right.$ as $\left({ }^{15} \mathrm{~N} /\right.$ total $\left.\left.\mathrm{N}\right) \times 100\right)$ with an Elemental Analyser (Thermo Electron FlashEA 1112) and subsequent isotope ratio mass spectrometry (Thermo Delta V - IRMS). For recent guidelines on stable isotope notations, see Coplen (2011). calculated according to Morris et al. (2013):

$$
x\left({ }^{15} N\right)_{\text {aq. } . N}=\frac{\left(\left[N H_{4}^{+}\right]_{\text {tracer }} x\left({ }^{15} N\right)_{\text {tracer }}\right)+\left(\left[N H_{4}^{+}\right]_{\text {water }} x\left({ }^{15} N\right)_{\text {initial }}\right)}{\left[N H_{4}^{+}\right]_{\text {tracer }}+\left[N H_{4}^{+}\right]_{\text {water }}}
$$

244 where $x\left({ }^{15} \mathrm{~N}\right)$ tracer is the atomic fraction of the added tracer $(0.98),\left[\mathrm{NH}_{4}{ }^{+}\right]_{\text {water }}$ is $20.7 \pm 3.1 \mu \mathrm{mol}$ $\mathrm{NH}_{4}{ }^{+} \mathrm{L}^{-1}$ (mean $\pm \mathrm{SE}$ ), and $\mathrm{x}\left({ }^{15} \mathrm{~N}\right)$ initial is the atomic fraction of the initial water column (assumed to 
246 reflect ${ }^{15} \mathrm{~N}$ of atmospheric $\left.\mathrm{N}, 3.7 \times 10^{-3}\right)$. To provide an estimate of the change in ${ }^{15} \mathrm{NH}_{4}{ }^{+}(\mu \mathrm{mol}-$

$\left.247 \mathrm{NH}_{4}{ }^{+} \mathrm{L}^{-1}\right)$ concentrations between runs (i.e., $\left[\mathrm{NH}_{4}{ }^{+}\right]$tracer$)$, mean ${ }^{15} \mathrm{NH}_{4}{ }^{+}$uptake rates $\left(\mu \mathrm{mol} \mathrm{g}{ }^{-1}(\mathrm{DM}) \mathrm{h}^{-1}\right)$

248 for each species were multiplied by their total estimated biomass $\left(\mathrm{g} \mathrm{DM} \mathrm{m}^{-2}\right)$ and by the incubation

249 time (6 hours). Given the large volume of the flume water, the estimated ${ }^{15} \mathrm{NH}_{4}{ }^{+}$tracer concentrations

250 remained high, between 8.0 and $8.8 \mu$ mol- $\mathrm{NH}_{4}{ }^{+} \mathrm{L}^{-1}$, corresponding to an increase in total water column

$251 \quad\left[\mathrm{NH}_{4}{ }^{+}\right]$of between $27.5 \%$ and $29.7 \%$.

252 To calculate the $\mathrm{NH}_{4}{ }^{+}$uptake rate $\left(V\right.$ in $\left.\mu \mathrm{mol} \mathrm{g}{ }^{-1}(\mathrm{DM}) \mathrm{h}^{-1}\right)$ of each sample, we followed the 253 equation in Morris et al. (2013):

$$
V=[N]_{D M_{-} \text {sample }} \cdot \frac{\left(x^{E}\left({ }^{15} N\right)_{D M_{-} \text {sample }}\right)}{\left(x\left({ }^{15} N\right)_{\text {aq.N }} \cdot \Delta t\right)}
$$

254 where $\mathrm{x}^{\mathrm{E}}\left({ }^{15} \mathrm{~N}\right)$ DM_sample is the ${ }^{15} \mathrm{~N}$ excess atom fraction, calculated as the difference between the atomic

255 fraction measured in the biomass of the sample after incubation $\left(x\left({ }^{15} \mathrm{~N}\right)\right.$ DM_sample $)$ and the background

$256{ }^{15} \mathrm{~N}$ abundance $\mathrm{x}\left({ }^{15} \mathrm{~N}\right)$ DM_nat.ab measured on five background specimens for each species $((3.70 \pm 0.008)$

$257 \times 10^{-3}$ for Callitriche, $(3.69 \pm 0.018) \times 10^{-3}$ for Groenlandia $) ; \Delta t(\mathrm{~h})$ is the incubation time $(6 \mathrm{~h})$ and

$258[\mathrm{~N}]_{\text {DM_sample }}$ is the $\mathrm{N}$ content of the dry biomass ( $\mu \mathrm{mol} \mathrm{g}^{-1}$ (dry mass $\left.(\mathrm{DM})\right)$ of each sample.

\section{Hydrodynamic measurements}

260 To test the relationship between hydrodynamic parameters and nutrient uptake, vertical profiles of

261 velocity were measured with a 3D acoustic Doppler velocimeter (ADV, Nortek) over $30 \mathrm{~s}$ at $10 \mathrm{~Hz}$.

262 Within each profile, velocity was measured at seven vertical locations at 2, 5, 10, 12, 15, 17 and $27 \mathrm{~cm}$

263 above the channel bed. The profiles were measured in the same streamwise and lateral locations as the

264 plant samples collected for nutrient uptake estimation, i.e. at 10\%, 50\% and $90 \%$ of the length of each

265 patch in the streamwise (x) direction, and at 0.15 and $0.25 \mathrm{~m}$ of the patch width in the spanwise (y) 
direction (Figure 2). To minimize interference by vegetation structures within the sampling volume of the ADV probe, the probe started at the lowest measuring point for each vertical profile within the vegetation. This prevented the canopy from being compressed as the probe moved towards the bed. To ensure that measurements were based on reliable data points, spikes and low-quality data points (i.e., correlation below the standard quality threshold of 70\%) were removed during post-processing. The height of the vegetation canopy in each location was measured with a ruler in $\mathrm{cm}$. The canopy height (h) was $0.17 \pm 0.08 \mathrm{~m}$ for Callitriche, and $0.07 \pm 0.01 \mathrm{~m}$ for Groenlandia (Table 1). Freshwater macrophytes are flexible, mesh-like structures (Sand-Jensen 2005), often with highly branched stems that get entangled in each other. Their flexibility and complex morphology make it very challenging to measure individual plant parameters like shoot height, frontal area, shoot density or average distance between shoots. Thus, the frontal area per water volume $\left(a, \mathrm{~m}^{-1}\right)$ and frontal area per bed area $(a h$, dimensionless) for the two species were calculated from water depth $(H)$ and canopy length $(l)$, width (y) and height $(h)$ measurements (Table 1$)$ through the following equations:

$$
\begin{aligned}
& a=\frac{h y}{H y l}=\frac{h}{H l} \\
& a h=\frac{h y}{y l}=\frac{h}{l}
\end{aligned}
$$

279 As the frontal area of flexible submerged macrophytes is highly variable (i.e. their shape and canopy height change with flow velocity; Sand-Jensen (2005)), the values are indicative for the incoming flow velocity of $0.24 \mathrm{~m} \mathrm{~s}^{-1}$ used in the experiments.

The instantaneous velocity $(u(t), v(t), w(t))$ measured in the streamwise, lateral, and vertical directions, respectively, were separated into time-averages $(\bar{U}, \bar{V}, \bar{W})$, and instantaneous turbulent fluctuations $\left(u^{\prime}(t), v^{\prime}(t), w^{\prime}(t)\right)$, e.g. as $u^{\prime}(t)=u(t)-\bar{U}$, and similarly for $v$ and $w$. The vertical average of the time-averaged velocity in the streamwise direction was used to calculate the depth- 
averaged velocity $\left.(<\overline{\mathrm{U}}\rangle, \mathrm{m} \mathrm{s}^{-1}\right)$ at each profile position. The Total Kinetic Energy (Total KE) per unit

287 mass is defined from the instantaneous velocities $(u, v, w)$, defined as:

$$
\text { Total } K E=\frac{1}{2}\left(\overline{u^{2}}+\overline{v^{2}}+\overline{w^{2}}\right)=\text { Turbulent } K E+\text { Mean } K E
$$

288 which can be partitioned into turbulent kinetic energy (Turbulent KE) and mean kinetic energy (Mean 289 KE), defined as (e.g. Kundu et al. (2004)):

$$
\begin{gathered}
\text { Turbulent } K E=\frac{1}{2}\left(\overline{u^{\prime 2}}+\overline{v^{\prime 2}}+\overline{w^{\prime 2}}\right) \\
\text { Mean } K E=\frac{1}{2}\left(\bar{U}^{2}+\bar{V}^{2}+\bar{W}^{2}\right)
\end{gathered}
$$

The Total Kinetic Energy $\left(\mathrm{m}^{2} \mathrm{~s}^{-2}\right)$ provides a better metric for the instantaneous velocity, because it reflects both the time-mean and turbulent fluctuations, and as such it is more relevant to boundary layer dynamics, especially in cases with low time-mean velocity but high Turbulent KE. periodically strip away the diffusive sub-layer, which, if frequent enough, will enhance flux to the plant surface (Koch 1994; Stevens and Hurd 1997; Huang et al. 2011).

Reynolds shear stress $\left(\tau_{\mathrm{xz}}, \mathrm{Pa}\right)$ at the top of the canopy at each location was calculated as:

$$
\tau_{x z}=-\rho \overline{u^{\prime}(t) w^{\prime}(t)}
$$

297 in which $\rho=1000 \mathrm{~kg} \mathrm{~m}^{-3}$ is the density of the flume water.

298 Volumetric flow rate of water through the patches $\left(\mathrm{Q}_{\mathrm{c}}, \mathrm{m}^{3} \mathrm{~s}^{-1}\right)$ was calculated as:

$$
Q_{c}=\sum_{0}^{h} Q_{i} \quad \text { and } \quad Q_{i}=y\left(h_{i}-h_{i-1}\right) \bar{u}_{h_{i}}
$$


299 in which $h$ is the canopy height, $Q_{i}$ the volumetric flow rate of water through the layer $\left(h_{i}-h_{i-1}\right), y$ is

300 the patch width $(0.3 \mathrm{~m})$ and $\bar{u}_{h_{i}}$ the double-averaged $u$ component (i.e., averaged in time and spatially

301 averaged in the two lateral positions) of the velocity at depth $h_{i}$.

\section{Measuring channel-scale patterns of ammonium uptake}

303 To investigate how the relationship between hydrodynamic parameters and ammonium uptake

304 develops at the scale of a whole channel, we tested the correlation between the total in-patch $\mathrm{NH}_{4}^{+}$

305 uptake rates and in-patch average hydrodynamic parameters (mean velocity, Turbulent KE and Total

306 KE). This allowed us to test whether spatial patch configurations that generated higher mean flow

307 velocity, Total KE or Turbulent KE levels within the canopies promoted higher uptake at the channel

308 scale. The total in-patch $\mathrm{NH}_{4}{ }^{+}$uptake rate for each configuration was calculated as the sum of the

309 uptake rates estimated in all sampling points ( $n=6$ per species; Figure 2$)$. This total uptake was used as

310 an estimate of channel-scale uptake, but is not necessarily a measure of total ammonium uptake rates

311 per biomass or aerial cover.

\section{Statistical analyses}

313 The flume incubation experiments yielded $n=30$ samples per experimental run (i.e. from $3 x$-positions

$314 \times 2 y$-positions $\times 5$ shoots per position) for each species, and two replicate runs were performed for

315 each configuration. As we were interested in differences in uptake rates among positions within

316 patches, the average uptake rates of the 5 shoots per each position were used in subsequent analyses.

317 Friedman's rank-sum test was used to test for the presence of trends in $\mathrm{NH}_{4}{ }^{+}$uptake rates from the

318 upstream to downstream positions along the patches in all replicate runs. The tests were run separately

319 for each species, both for the centerline and for the edge measurement points. To account for the fact

320 that within-patch measurements were not independent from each other, statistical differences in $\mathrm{NH}_{4}^{+}$

321 uptake rates for each species under four flume spatial configurations were tested using nested ANOVA 
322 (with replicate run as a nested factor within the configuration treatment). As Friedman's rank-sum tests

323 showed no significant trend in ammonium uptake neither along the centerline nor along the edge

324 positions within patches, measurement position was considered a random effect in the model. The data

325 were log-transformed to meet the ANOVA test assumptions of normality and homogeneity of variance.

326 Pearson's correlation coefficient was used to test for significant correlation between $\mathrm{NH}_{4}{ }^{+}$uptake rates

$327\left(\mu \mathrm{mol} \mathrm{g}{ }^{-1}(\mathrm{DM}) \mathrm{h}^{-1}\right)$ and hydrodynamic parameters (depth-averaged velocity $\langle\overline{\mathrm{U}}\rangle\left(\mathrm{m} \mathrm{s}^{-1}\right)$; Reynolds

328 shear stress $\tau_{\mathrm{xz}}(\mathrm{Pa})$; Turbulent $\mathrm{KE}\left(\mathrm{m}^{2} \mathrm{~s}^{-2}\right)$; Total $\mathrm{KE}\left(\mathrm{m}^{2} \mathrm{~s}^{-2}\right)$; and volumetric flow rate, $\mathrm{Q}_{\mathrm{c}}\left(\mathrm{m}^{3} \mathrm{~s}^{-1}\right)$,

329 and between channel total $\mathrm{NH}_{4}{ }^{+}$uptake rates and average hydrodynamic parameters within both

330 species patches (mean velocity, Turbulent KE and Total KE). $P$ values of less than or equal to 0.05

331 were considered to be significant. All statistical analyses were performed in R 3.1.2 (R Core Team

332 2015).

333 Results

$334 \quad$ Relationship between canopy hydrodynamic parameters and nutrient uptake

335 We found that the two macrophyte species affected each other's ammonium uptake rates by altering

336 mean flow velocity $(\langle\overline{\mathrm{U}}\rangle$ ) and turbulence (Turbulent KE). Ammonium uptake rates depended on either

337 mean flow velocity (Callitriche) or turbulence (Groenlandia), but Total Kinetic Energy (Total KE) was

338 the single best descriptor of uptake rates for both species (Figure 3; Table 2). Specifically, $\mathrm{NH}_{4}^{+}$uptake $^{-}$

339 rates for the sparse Groenlandia were significantly correlated with Turbulent $\mathrm{KE}(r=0.68, p<0.001)$,

340 but not with mean flow velocity $(r=-0.20, p=0.35)$ (Figure 3; Table 2). The opposite was true for the

341 dense Callitriche: uptake rates were significantly correlated with mean flow velocity $(r=0.42, p=$

$3420.04)$, but not with Turbulent KE $(r=0.34, p=0.1)$ (Figure 3; Table 2). However, Total KE, which is

343 more representative of the instantaneous velocity, described uptake for both species $(r=0.79, p<0.001$ 
344 for Groenlandia; $r=0.45, p=0.03$ for Callitriche; $r=0.54, p<0.001$ for both species together)

345 (Figure 3A; Table 2). No significant relationship was found between ammonium uptake rates and either 346 Reynolds shear stress or $\mathrm{Q}_{\mathrm{c}}$ (Table 2).

\section{Effects of patch spatial configurations on nutrient uptake}

348 When located upstream, the dense Callitriche patch increased turbulence and thereby enhanced the uptake of resources by the sparse Groenlandia patch located downstream. The ammonium uptake rates were influenced by both macrophyte species and spatial patch configuration (order and alignment). Importantly, the Dense-Sparse (D-S) configurations led to higher uptake rates for both species. The $\mathrm{NH}_{4}{ }^{+}$uptake rates for the sparse Groenlandia were $2.63 \pm 1.33 \mu \mathrm{mol} \mathrm{g}{ }^{-1}(\mathrm{DM}) \mathrm{h}^{-1}$, almost double than for the dense Callitriche $\left(1.44 \pm 0.78 \mu \mathrm{mol} \mathrm{g}^{-1}(\mathrm{DM}) \mathrm{h}^{-1}\right)$. Testing for the presence of patterns in $\mathrm{NH}_{4}^{+}$ uptake rates from upstream to downstream within the patches showed no significant trend in uptake rates along the patch centerline (Friedman $\chi_{2}^{2}=3, p=0.22$ for Callitriche; Friedman $\chi_{2}^{2}=1, p=0.60$ for Groenlandia), nor along the patch edge (Friedman $\chi_{2}^{2}=0.25, p=088$ for Callitriche; Friedman $\chi_{2}^{2}$ $=3, p=0.22$ for Groenlandia). This indicates there was no significant pattern in stable isotope concentration from upstream to downstream within patches. affected uptake rates for both the sparse Groenlandia (nested ANOVA, $F_{3,4}=6.87, p=0.04$ ) and the dense Callitriche (nested ANOVA, $F_{3,4}=12.57, p=0.017$; Figure 4). We generally found that when the denser species (Callitriche) was located upstream of the sparser one (Groenlandia), ammonium uptake rates for both species increased significantly, compared to patch configurations in the SparseDense order (Figure 4). This significant increase in uptake rates was related to the hydrodynamic effects of different configurations, and particularly the traits of Callitriche (i.e. density and canopy height, which blocks a larger fraction of flow depth). When the dense patch of Callitriche was 
upstream, it generated higher Turbulent KE that influenced the downstream patch of Groenlandia

368 (Figure 2), enhancing its uptake rates (Figure 3). Also, when the dense Callitriche was upstream, its leading edge was exposed to higher mean velocity compared to when it was trailing behind the sparse patch (Figure 2), thereby increasing its uptake rates (Figure 3). Specifically, for the dense Callitriche, uptake rates within the Sparse-Dense order were higher in the staggered than in the aligned configuration (Tukey's HSD, $z=-2.66, p<0.05$ ). In the Dense-Sparse configurations, no significant difference in uptake rates was found between the staggered or aligned arrangement (Tukey's HSD, $z=$ $-0.50, p>0.05)$. However, uptake rates were significantly higher in the Dense-Sparse staggered configuration than in both the S-D configurations (Tukey's HSD, $z=-2.83, p<0.05$ ). For the sparse Groenlandia, uptake rates within the Sparse-Dense aligned and staggered configuration were not significantly different from each other (Tukey's HSD, $z=-1.92, p<0.05$ ). In the Dense-Sparse configurations, no significant difference in uptake rates was found between the staggered or aligned arrangement (Tukey's HSD, $z=0.51, p>0.05$ ). Uptake rates in the $\mathrm{D}-\mathrm{S}$ aligned configuration were significantly higher than in the S-D aligned configuration (Tukey's HSD, $z=-2.69, p<0.05$ ), but were not significantly different from the S-D staggered case (Tukey’s HSD, $z=-0.77, p>0.05$ ). within the patches promoted higher total uptake at the channel scale (Figure 5). Testing for the hydrodynamic parameter-uptake relationships at the channel scale revealed a significant positive relationship between the in-patch Total Kinetic Energy (average of both patches in each configuration) and the channel total ammonium uptake $(r=0.98, p=0.01$; Figure 5). Channel total ammonium uptake was also significantly related to in-patch Turbulent KE $(r=0.97, p=0.03)$, but not to mean flow velocity $(r=0.93, p=0.07)$.

\section{Discussion}


The interaction between vegetation and hydrodynamics regulates important ecological processes such as nutrient delivery and uptake by aquatic plants, which are crucial for community primary productivity (Thomas et al. 2000; Cornelisen and Thomas 2006; Morris et al. 2008). We found that, by generating turbulence, dense macrophyte patches facilitate resource uptake by neighboring sparse patches. Flume measurements showed that the dense Callitriche had a strong hydrodynamic effect, creating highturbulence regions in its wake that facilitated nutrient uptake by the sparse Groenlandia, which had a weaker hydrodynamic effect. While the sparse vegetation benefited from the high turbulence generated in the wake of a dense patch, the dense vegetation benefited from being located at a leading edge, where it was exposed to higher mean velocity, compared to when it was located downstream of another patch (Figure 2; Figure 6). We identified Total Kinetic Energy as the best descriptor of the nutrient removal capacity of streams, especially in heterogeneous multi-species communities. Overall, spatial configurations that lead to higher Total Kinetic Energy within the patches were the ones that led to higher total ammonium uptake. Hence, our results highlight the importance of turbulence as an agent of interaction between different species. Moreover, this study suggests that accounting for interactions between heterogeneous, multi-specific patchy vegetation is crucial to understand aquatic ecosystem functions such as nitrogen retention.

\section{Implications of resource uptake in mono- and multi-species communities}

Previous studies of macrophytes generally found that nutrient uptake rates increased with mean flow velocity (Cornelisen and Thomas 2006; Bal et al. 2013). Morris et al. (2008) found that Turbulent KE was associated with spatial variation in uptake, and volumetric flow rate explained differences in uptake between contrasting species. Yet, in our study, neither of these traditional hydrodynamic parameters could accurately describe uptake rates for both species. However, direct comparison with these previous studies is limited to some extent by differences in treatments. While previous 
413 experiments tested the effect of changing bulk flow velocity on uptake rates, we tested a single bulk

414 flow velocity that represented the average flow conditions in the study sites where Callitriche and

415 Groenlandia coexist. We thus focused on within-patch flow variability, and how the interaction

416 between the two species was determined by their spatial arrangement and flow modification. Using a

417 single bulk flow condition resulted in a relatively narrow range of velocities experienced by the sparse

418 Groenlandia $\left(0.13-0.27 \mathrm{~m} \mathrm{~s}^{-1}\right)$, whereas the flow alteration ability of the dense Callitriche led to a

419 higher flow variability $\left(0.002-0.24 \mathrm{~m} \mathrm{~s}^{-1}\right)$. This factor may partly explain the lack of significant

420 correlation between flow velocity and uptake rates for Groenlandia. Further research is needed to test

421 how changes in mean flow conditions affect the interaction between the two species.

We identified Total Kinetic Energy as the parameter that explained most of the variability in

uptake rates for both species. To our knowledge, this parameter has not been related before to nutrient

uptake rates by aquatic vegetation. Previous studies have suggested that Turbulent KE may influence

nutrient uptake (Anderson and Charters 1982; Koch 1994), and the total energy parameter captures this

influence. Specifically, when Turbulent KE is weak, flux is controlled by the time-mean diffusive sub-

layer thickness, which is a function of the time-mean velocity (e.g. Hansen et al. (2011); Rominger and

Nepf (2014); Lei and Nepf (2016)). However, when the Turbulent KE is high, periodic disturbances of

the diffusive sub-layer by the turbulence can create instantaneously higher concentration gradients at the surface and, thus, higher flux (e.g. Stevens and Hurd (1997); Huang et al. (2011); Rominger and Nepf (2014)). By reflecting the magnitude of both the time-mean velocity and Turbulent KE, the total kinetic energy captures both regimes of flux. The Total Kinetic Energy is particularly suitable in heterogeneous systems where upstream Turbulent KE generation (e.g. by larger, denser patches) can influence flux downstream, i.e. the Turbulent KE is not locally generated and thus uncorrelated with the local time-mean velocity. In the dense Callitriche patches, the canopy is often too dense for turbulence to form within the patch or to penetrate from the free stream. Under these low Turbulent KE 
conditions, the flux is correlated by the local time-mean velocity, which sets the scale of the diffusive sub-layer. For the sparse Groenlandia, time-mean flow velocity is relatively constant in the canopy and we found no correlation between within-patch flow variations and uptake rates. However, the withinpatch Turbulent KE is elevated both by local stem generation and the penetration of turbulence generated upstream. Under these high Turbulent KE conditions, the uptake rates have a high correlation with the Turbulent KE intensity. In landscapes made up of patches of different species, regions with flux controlled by $\langle\overline{\mathrm{U}}\rangle$ and regions controlled by Turbulent KE are heterogeneously distributed, so that neither $\langle\overline{\mathrm{U}}\rangle$ nor Turbulent KE can capture the channel-scale nutrient uptake. Because it can describe both regions of low Turbulent KE (uptake controlled by mean velocity) and high Turbulent KE (uptake controlled by Turbulent KE intensity), we propose Total Kinetic Energy as a useful parameter to describe nutrient uptake capacity in heterogeneous landscapes, which could be used to estimate ecosystem services of nutrient retention by vegetation.

In contrast to the findings of Morris et al. (2008) and Bal et al. (2013), we did not find a significant relationship between ammonium uptake rates and volumetric flow rate, likely because the two species have different flexibility and density traits that affect patch compression (Table 1). The canopies of the two species, and the relative importance of flow velocity and turbulence within them, are consistent with the sparse and dense canopy regimes described in Nepf (2012). As expected for a dense canopy condition ( $a h>0.1)$, canopy-scale turbulence in Callitriche is generated at the top of the canopy and can be transported downstream, while stem-scale turbulence is much smaller. Instead, Groenlandia is representative of a sparse canopy condition $(a h<0.1)$, where stem-scale turbulence is generated within the canopy, but the velocity profile remains logarithmic. heterogeneity in influencing species interactions and the nutrient uptake capacity of the landscape. The generation of turbulence by a Callitriche patch led to a $59 \%$ nutrient uptake enhancement effect on 
461 Groenlandia, increasing it from an ammonium uptake rate of $2.03 \pm 0.85 \mu \mathrm{mol} \mathrm{g}^{-1}(\mathrm{DM}) \mathrm{h}^{-1}$ in the

462 Sparse-Dense configurations (where Groenlandia was not exposed to turbulence generation by

463 Callitriche), to $3.23 \pm 0.37 \mu \mathrm{mol} \mathrm{g}^{-1}(\mathrm{DM}) \mathrm{h}^{-1}$ in the Dense-Sparse configurations (where it was

464 exposed to turbulence generation by Callitriche). These findings are in line with a field study showing

465 that spatial heterogeneity, created by the interaction of canopy morphology, sediment topography and

466 hydrodynamics, controlled nutrient transport and uptake rates in a patchy seagrass landscape (Morris et

467 al. 2013). While we found clear effects of the upstream-downstream patch arrangement on ammonium

468 uptake rates, we only observed a significant effect of patch arrangement (staggered vs. aligned) in the

469 Sparse-Dense configurations. Vegetation patches in the flume experiment did not span the whole width

470 of the channel, leading to water flow deflection and acceleration around the lateral edges of patches.

471 This flow acceleration effect around the patches is in general agreement with experimental evidence in

472 field and laboratory studies (Vandenbruwaene et al. 2011; Schoelynck et al. 2012; Bouma et al. 2013),

473 suggesting that the conclusions of this work could be generally applied to field conditions. In addition

474 to the role of spatial configuration, it is likely that the distance between the patches governs the

475 intensity of the interaction between them. The stronger interactions between patches likely occur when

476 the distance between them is less than the wake length of the upstream patch (Folkard 2005). It might

477 be expected that the wake length is in turn related to patch density, because density determines at what

478 distances the patch effects will dissipate (Zong and Nepf 2012). Further studies should be undertaken to

479 investigate the detailed hydrodynamic consequences of different spatial patch configurations, testing

480 for the effects of a wider range of distances and its interactive effect with patch density.

$481 \quad$ Turbulence-mediated species interactions: implications for species distributions and nutrient load

482 reduction 
483 The study of turbulence-mediated interactions between macrophyte species suggests a possible 484 mechanism behind the co-occurrence of Groenlandia patches around Callitriche in the field. Recently, 485 it has been shown that Groenlandia shoots grow better around Callitriche patches than on bare, 486 unvegetated sediment (Cornacchia et al. 2018). The wake of the Callitriche patches is both a high487 turbulence and low-velocity region (Sand-Jensen 1998). Thus, a combination of enhanced resource 488 uptake by turbulence, and reduced biomass losses by flow velocity, might be the conditions behind the 489 improved growth rates of Groenlandia plants around Callitriche patches. As the sparse Groenlandia tends to surround the dense Callitriche patches in regularly spaced aggregations every $8 \mathrm{~m}$ (Cornacchia et al. 2018), the interaction between the two species might enhance the overall nutrient removal capacity of the river. The facilitative effect of Callitriche on Groenlandia could switch to competition, as the high biomass Callitriche might have a competitive advantage by reducing resource availability for the sparser species (Groenlandia). On the other hand, competition could be lessened through root uptake from the nutrient pool in the sediment. The balance between facilitation and competition can be clarified by considering additional variables such as root uptake and nutrient availability in the water column and in sediment. Moreover, care must be taken when upscaling the relationship between hydrodynamics and resource uptake at the channel scale. In our incubations, we focused only on uptake rates of a single nutrient (ammonium), which is energetically less costly, but some species might invest in nitrate uptake. This is an interesting aspect that should be explored in future studies of channel-scale nitrogen uptake by vegetation. As a future perspective, we might be able to use the knowledge on these types of species interactions as tools to enhance restoration success of degraded (eutrophic) sites.

\section{References}


Adhitya, A. and others 2014. Comparison of the influence of patch-scale and meadow-scale characteristics on flow within seagrass meadows: a flume study. Marine Ecology Progress Series 516: 49-59.

Anderson, S. M., and A. Charters. 1982. A fluid dynamics study of seawater flow through Gelidium nudifrons. Limnology and Oceanography 27: 399-412.

Bal, K. D. and others 2013. Influence of hydraulics on the uptake of ammonium by two freshwater plants. Freshwater Biology 58: 2452-2463.

Bouma, T. and others 2005. Trade-offs related to ecosystem engineering: A case study on stiffness of emerging macrophytes. Ecology 86: 2187-2199.

Bouma, T., M. Friedrichs, B. Van Wesenbeeck, S. Temmerman, G. Graf, and P. Herman. 2009. Density-dependent linkage of scale-dependent feedbacks: A flume study on the intertidal macrophyte Spartina anglica. Oikos 118: 260-268.

Bouma, T. and others 2013. Organism traits determine the strength of scale-dependent bio-geomorphic feedbacks: A flume study on three intertidal plant species. Geomorphology 180: 57-65.

Bouma, T. and others 2007. Spatial flow and sedimentation patterns within patches of epibenthic structures: Combining field, flume and modelling experiments. Continental Shelf Research 27: 1020-1045.

Chen, Z., C. Jiang, and H. Nepf. 2013. Flow adjustment at the leading edge of a submerged aquatic canopy. Water Resources Research 49: 5537-5551.

Chen, Z., A. Ortiz, L. Zong, and H. Nepf. 2012. The wake structure behind a porous obstruction and its implications for deposition near a finite patch of emergent vegetation. Water Resources Research 48.

Coplen, T. B. 2011. Guidelines and recommended terms for expression of stable-isotope-ratio and gasratio measurement results. Rapid communications in mass spectrometry 25: 2538-2560. 
529 Corenblit, D. and others 2011. Feedbacks between geomorphology and biota controlling Earth surface 530 processes and landforms: a review of foundation concepts and current understandings. Earth$531 \quad$ Science Reviews 106: 307-331.

532 Cornacchia, L., J. van de Koppel, D. van der Wal, G. Wharton, S. Puijalon, and T. J. Bouma. 2018. 533 Landscapes of facilitation: how self-organized patchiness of aquatic macrophytes promotes diversity in streams. Ecology.

Cornelisen, C. D., and F. I. Thomas. 2004. Ammonium and nitrate uptake by leaves of the seagrass Thalassia testudinum: impact of hydrodynamic regime and epiphyte cover on uptake rates. Journal of Marine Systems 49: 177-194.

---. 2006. Water flow enhances ammonium and nitrate uptake in a seagrass community. Marine Ecology Progress Series 312: 1-13.

Cotton, J., G. Wharton, J. Bass, C. Heppell, and R. Wotton. 2006. The effects of seasonal changes to in-stream vegetation cover on patterns of flow and accumulation of sediment. Geomorphology 77: $320-334$.

Dawson, F., and W. Robinson. 1984. Submerged macrophytes and the hydraulic roughness of a lowland chalkstream. Verhandlung Internationale Vereinigung Limnologie 22.

Dietrich, W. E., and J. T. Perron. 2006. The search for a topographic signature of life. Nature 439: 411418.

Folkard, A. M. 2005. Hydrodynamics of model Posidonia oceanica patches in shallow water. Limnology and oceanography 50: 1592-1600.

Follett, E. M., and H. M. Nepf. 2012. Sediment patterns near a model patch of reedy emergent vegetation. Geomorphology 179: 141-151. 
551 Fonseca, M. S., J. C. Zieman, G. W. Thayer, and J. S. Fisher. 1983. The role of current velocity in structuring eelgrass (Zostera marina L.) meadows. Estuarine, Coastal and Shelf Science 17: $367-380$.

Gambi, M. C., A. R. Nowell, and P. A. Jumars. 1990. Flume observations on flow dynamics in Zostera marina (eelgrass) beds. Marine ecology progress series: 159-169.

Hansen, A. T., M. Hondzo, and C. L. Hurd. 2011. Photosynthetic oxygen flux by Macrocystis pyrifera: a mass transfer model with experimental validation. Marine Ecology Progress Series 434: 4555.

Haynes, R., and K. M. Goh. 1978. Ammonium and nitrate nutrition of plants. Biological Reviews 53: 465-510.

Huang, I., J. Rominger, and H. Nepf. 2011. The motion of kelp blades and the surface renewal model. Limnology and Oceanography 56: 1453-1462.

Järvelä, J. 2005. Effect of submerged flexible vegetation on flow structure and resistance. Journal of Hydrology 307: 233-241.

Jones, C. G., J. H. Lawton, and M. Shachak. 1994. Organisms as ecosystem engineers, p. 130-147. Ecosystem management. Springer.

Koch, E. 1994. Hydrodynamics, diffusion-boundary layers and photosynthesis of the seagrasses Thalassia testudinum and Cymodocea nodosa. Marine Biology 118: 767-776.

Kouwen, N., and T. E. Unny. 1973. Flexible roughness in open channels. Journal of the Hydraulics Division 99.

Kundu, P. K., I. M. Cohen, and H. H. Hu. 2004. Fluid mechanics, 6th ed. ISBN: 978-0-12-405935-1. Elsevier Press, Boston.

Lei, J., and H. Nepf. 2016. Impact of current speed on mass flux to a model flexible seagrass blade. Journal of Geophysical Research: Oceans 121: 4763-4776. 
575 Leonard, L. A., and M. E. Luther. 1995. Flow hydrodynamics in tidal marsh canopies. Limnology and $576 \quad$ oceanography 40: 1474-1484.

577 Levi, P. S. and others 2015. Macrophyte complexity controls nutrient uptake in lowland streams.

$578 \quad$ Ecosystems 18: 914-931.

579 Madsen, J. D., P. A. Chambers, W. F. James, E. W. Koch, and D. F. Westlake. 2001. The interaction

580 between water movement, sediment dynamics and submersed macrophytes. Hydrobiologia 444:

$581 \quad 71-84$

582 Madsen, T. V., and N. Cedergreen. 2002. Sources of nutrients to rooted submerged macrophytes

583 growing in a nutrient-rich stream. Freshwater Biology 47: 283-291.

584 Meire, D. W., J. M. Kondziolka, and H. M. Nepf. 2014. Interaction between neighboring vegetation

585 patches: Impact on flow and deposition. Water Resources Research 50: 3809-3825.

586 Morris, E. P., G. Peralta, F. G. Brun, L. Van Duren, T. J. Bouma, and J. L. Perez-Llorens. 2008.

587 Interaction between hydrodynamics and seagrass canopy structure: Spatially explicit effects on

588 ammonium uptake rates. Limnology and Oceanography 53: 1531-1539.

589 Morris, E. P. and others 2013. The role of hydrodynamics in structuring in situ ammonium uptake

590 within a submerged macrophyte community. Limnology and Oceanography: Fluids and

$591 \quad$ Environments 3: 210-224.

592 Nepf, H. 1999. Drag, turbulence, and diffusion in flow through emergent vegetation. Water resources research 35: 479-489.

594 Nepf, H., and E. Vivoni. 2000. Flow structure in depth-limited, vegetated flow. Journal of Geophysical Research: Oceans 105: 28547-28557.

Nepf, H. M. 2012. Flow and transport in regions with aquatic vegetation. Annual Review of Fluid Mechanics 44: 123-142. 
598 Peralta, G., L. Van Duren, E. Morris, and T. Bouma. 2008. Consequences of shoot density and stiffness

599 for ecosystem engineering by benthic macrophytes in flow dominated areas: a hydrodynamic

$600 \quad$ flume study. Marine Ecology Progress Series 368: 103-115.

601 R Core Team. 2015. R: A Language and Environment for Statistical Computing (Version 3.1. 2): R

602 Foundation for Statistical Computing. Vienna, Austria. URL http://www.R-project.org.

603 Rominger, J. T., and H. M. Nepf. 2014. Effects of blade flexural rigidity on drag force and mass

604 transfer rates in model blades. Limnology and Oceanography 59: 2028-2041.

605 Sand-Jensen, K. 1998. Influence of submerged macrophytes on sediment composition and near-bed

606 flow in lowland streams. Freshwater Biology 39: 663-679.

607 Sand-Jensen, K., and T. Vindbœk Madsen. 1992. Patch dynamics of the stream macrophyte, Callitriche $608 \quad$ cophocarpa. Freshwater Biology 27: 277-282.

609 Sand-Jensen, K. 2005. Aquatic plants are open flexible structures-a reply to Sukhodolov. Freshwater

$610 \quad$ Biology 50: 196-198.

611 Schoelynck, J., T. De Groote, K. Bal, W. Vandenbruwaene, P. Meire, and S. Temmerman. 2012. Self-

612 organised patchiness and scale-dependent bio-geomorphic feedbacks in aquatic river vegetation.

$613 \quad$ Ecography 35: 760-768.

614 Schulz, M., H.-P. Kozerski, T. Pluntke, and K. Rinke. 2003. The influence of macrophytes on

615 sedimentation and nutrient retention in the lower River Spree (Germany). Water Research 37:

$616 \quad 569-578$.

617 Sheldon, R. B., and C. W. Boylen. 1977. Maximum depth inhabited by aquatic vascular plants.

618 American midland naturalist: 248-254.

619 Stevens, C. L., and C. L. Hurd. 1997. Boundary-layers around bladed aquatic macrophytes.

$620 \quad$ Hydrobiologia 346: 119-128. 
621 Thomas, F. I., C. D. Cornelisen, and J. M. Zande. 2000. Effects of water velocity and canopy morphology on ammonium uptake by seagrass communities. Ecology 81: 2704-2713.

Vandenbruwaene, W. and others 2011. Flow interaction with dynamic vegetation patches: Implications for biogeomorphic evolution of a tidal landscape. Journal of Geophysical Research: Earth Surface 116.

Weitzman, J. S., R. B. Zeller, F. I. Thomas, and J. R. Koseff. 2015. The attenuation of current-and wave-driven flow within submerged multispecific vegetative canopies. Limnology and Oceanography 60: $1855-1874$.

Wharton, G. and others 2006. Macrophytes and suspension-feeding invertebrates modify flows and fine sediments in the Frome and Piddle catchments, Dorset (UK). Journal of Hydrology 330: 171184.

Zong, L., and H. Nepf. 2012. Vortex development behind a finite porous obstruction in a channel. Journal of Fluid Mechanics 691: 368-391.

\section{Acknowledgments}

The authors gratefully acknowledge Bert Sinke, Lennart van IJzerloo, Lowie Haazen and Jeroen van Dalen for their technical assistance in the flume. We thank Peter van Breugel and members of the analytical lab of NIOZ-Yerseke for the stable isotope analyses. We thank Siebren Wezenberg for helping during the experiments. This work was supported by the Research Executive Agency, through the 7th Framework Programme of the European Union, Support for Training and Career Development of Researchers (Marie Curie - FP7-PEOPLE-2012-ITN), which funded the Initial Training Network (ITN) HYTECH 'Hydrodynamic Transport in Ecologically Critical Heterogeneous Interfaces', N.316546. Data associated with this study is available from 4TU.Centre for Research Data at: 


\section{$644 \quad$ Figure legends}

645 Figure 1: (A) Natural patches of Callitriche and (B) Groenlandia in the field. (C, D) Lateral view of the 646 two patches, with the black outline indicating canopy height at increasing distance from the patch 647 leading edge.

648 Figure 2: Schematic diagram of the four spatial configurations of aquatic macrophytes in the test 649 section of the flume. Light green indicates patches of Groenlandia (sparse canopy), and dark green indicates patches of Callitriche (dense canopy). Diagonal lines indicate the boxes in which plants were rooted. Black circles are locations of plant specimens removed after the incubations experiments for assessment of $\mathrm{NH}_{4}^{+}$uptake rates, and of Acoustic Doppler Velocimeter (ADV) profile measurements. Numbers indicate mean $( \pm \mathrm{SE})$ water velocity $\overline{\mathrm{U}}\left(\mathrm{m} \mathrm{s}^{-1}\right)$ and Turbulent Kinetic Energy (Turbulent KE) $654\left(\mathrm{~m}^{2} \mathrm{~s}^{-2}\right)$ within each species patch.

Figure 3: Scatter plots of $\mathrm{NH}_{4}^{+}$uptake rates $\left(\mu \mathrm{mol} \mathrm{g}{ }^{-1}(\mathrm{DM}) \mathrm{h}^{-1}\right.$ ) against Total Kinetic Energy (Total KE) $\left(\mathrm{m}^{2} \mathrm{~s}^{-2}\right)$, depth-averaged velocity $<\overline{\mathrm{U}}>\left(\mathrm{m} \mathrm{s}^{-1}\right)$ and Turbulent Kinetic Energy (Turbulent KE) $\left(\mathrm{m}^{2} \mathrm{~s}^{-}\right.$ ${ }^{2}$ ) for the sparse Groenlandia (black circles) and the dense Callitriche (white diamonds). Black lines are linear regression lines for the Groenlandia (solid line) and Callitriche (dotted line) data separately and represent significant relationships $(\mathrm{p} \leq 0.05)$.

660 Figure 4: Boxplots of the distribution of $\mathrm{NH}_{4}{ }^{+}$uptake rates $\left(\mu \mathrm{mol} \mathrm{g}{ }^{-1}(\mathrm{DM}) \mathrm{h}^{-1}\right)$ within patches of the dense Callitriche (a) and the sparse Groenlandia (b) in each spatial configuration (S indicating sparse vegetation, D indicating dense vegetation, see Figure 2). Letters denote significant differences (Tukey’s HSD, $\mathrm{p}<0.05)$.

664 Figure 5: Scatter plots of channel total $\mathrm{NH}_{4}{ }^{+}$uptake rates $\left(\mu \mathrm{mol} \mathrm{g} \mathrm{g}^{-1}(\mathrm{DM}) \mathrm{h}^{-1}\right)$ in each spatial 665 configuration against Total Kinetic Energy $\left(\mathrm{m}^{2} \mathrm{~s}^{-2}\right)$ averaged within patches of Callitriche and 
666 Groenlandia in each spatial configuration (S indicating sparse vegetation, D indicating dense

667 vegetation, see Figure 2). Error bars represent standard error of the mean.

668 Figure 6: Schematized drawing of the effects of multispecific spatial patchiness on hydrodynamics and 669 nutrient uptake rates. In Sparse-Dense configurations (a), the sparse vegetation is exposed to high mean 670 flow but low turbulence, and does not benefit from being located at the leading edge. Similarly, the 671 dense vegetation is exposed to low mean flow speed due to sheltering by the patch upstream, and hence 672 has lower uptake rates. Instead, in Dense-Sparse configurations (b), uptake rates of both species are 673 higher: the dense vegetation benefits from being at the leading edge and exposed to high mean flow 674 speed (which increases uptake rates); at the same time, the sparse vegetation benefits from the high 675 turbulence created in the wake of the dense patch.

676

677

678 


\section{Tables}

680 Table 1 Summary of patch characteristics [mean \pm SD $(n)$ ] of the two species at the incoming flow

681 velocity of $0.24 \mathrm{~m} \mathrm{~s}^{-1}$ used in the flume experiments: biomass ( $\mathrm{g} \mathrm{DW} \mathrm{m}{ }^{-2}$; measured), canopy height ( $h$,

$682 \mathrm{~m}$; measured), frontal area per water volume ( $a, \mathrm{~m}^{-1}$; calculated from Eq. 3), frontal area per bed area

683 (ah, dimensionless; calculated from Eq. 4).

\begin{tabular}{|l|l|c|c|c|}
\hline & \multicolumn{1}{|c|}{$\begin{array}{c}\text { Biomass } \\
\left(\mathrm{g} \mathrm{DW} \mathrm{m}^{-2}\right)\end{array}$} & $\begin{array}{c}\text { Canopy height } \\
(h, \mathrm{~m})\end{array}$ & $\begin{array}{c}\text { Frontal area per } \\
\text { water volume } \\
\left(a, \mathrm{~m}^{-1}\right)\end{array}$ & $\begin{array}{c}\text { Frontal area per bed } \\
\text { area } \\
(\mathrm{ah}, \text { dimensionless })\end{array}$ \\
\hline Groenlandia & $97 \pm 28(3)$ & $0.070 \pm 0.010(9)$ & $0.090 \pm 0.007(2)$ & $0.031 \pm 0.003(2)$ \\
\hline Callitriche & $318 \pm 67(3)$ & $0.170 \pm 0.080(5)$ & $0.571 \pm 0.101(2)$ & $0.200 \pm 0.035(2)$ \\
\hline
\end{tabular}

684 
685 Table 2 Pearson's correlation coefficients between $\mathrm{NH}_{4}^{+}$uptake rates $\left(\mu \mathrm{mol} \mathrm{g} \mathrm{g}^{-1}(\mathrm{DM}) \mathrm{h}^{-1}\right)$ and canopy 686 height $(\mathrm{cm})$ or hydrodynamic parameters (depth-averaged velocity $<\overline{\mathrm{U}}>\left[\mathrm{m} \mathrm{s}^{-1}\right]$; Reynolds shear stress $687 \tau_{x z}[\mathrm{~Pa}]$; Turbulent Kinetic Energy (Turbulent KE) $\left[\mathrm{m}^{2} \mathrm{~s}^{-2}\right]$; Total Kinetic Energy (Total KE) $\left[\mathrm{m}^{2} \mathrm{~s}^{-2}\right]$ 688 and canopy water flow $Q_{c}\left[\mathrm{~m}^{3} \mathrm{~s}^{-1}\right]$ ) for Groenlandia, Callitriche and both species considered together. 689 Correlation coefficients in bold are significant at $p \leq 0.05$.

\begin{tabular}{|l|c|c|c|}
\hline & Groenlandia $(n=24)$ & Callitriche $(n=24)$ & All $(n=48)$ \\
\hline Height & 0.17 & -0.18 & $\mathbf{- 0 . 3 0}$ \\
\hline$<\overline{\mathrm{U}}>$ & -0.20 & $\mathbf{0 . 4 2}$ & $\mathbf{0 . 4 0}$ \\
\hline$\tau_{\mathrm{xz}}$ & 0.01 & -0.18 & 0.03 \\
\hline Turbulent KE & $\mathbf{0 . 6 8}$ & 0.34 & $\mathbf{0 . 5 3}$ \\
\hline Total KE & $\mathbf{0 . 7 9}$ & $\mathbf{0 . 4 5}$ & $\mathbf{0 . 5 4}$ \\
\hline $\mathrm{Q}_{\mathrm{c}}$ & -0.07 & 0.19 & -0.09 \\
\hline
\end{tabular}

690

691 


\section{Figures}

Callitriche

(dense species)

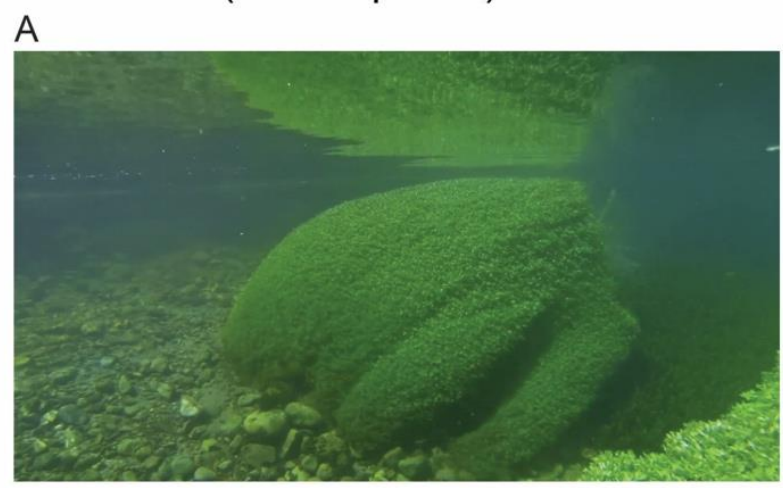

C

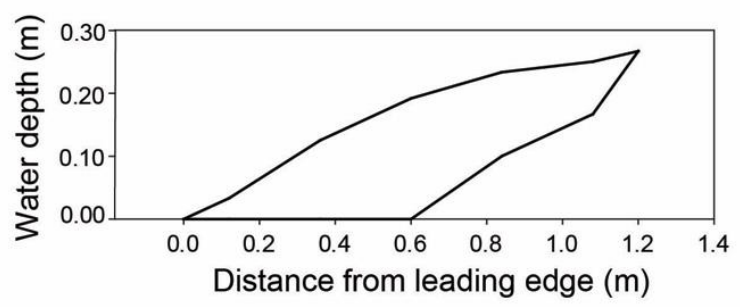

Groenlandia

(sparse species)

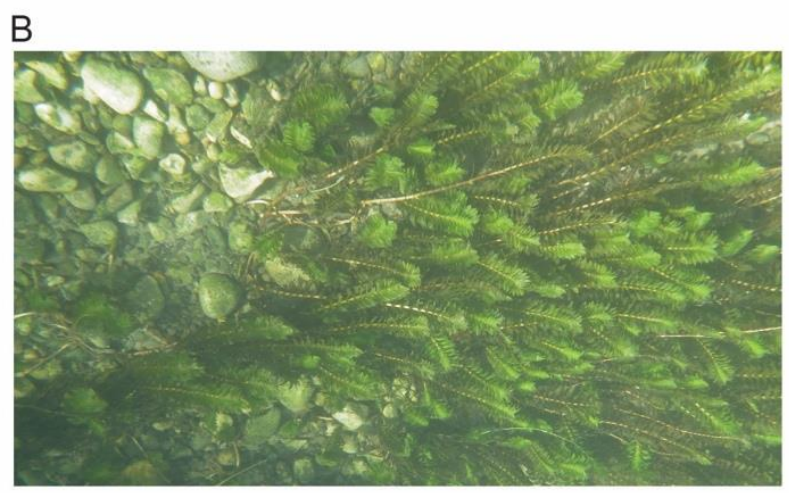

D

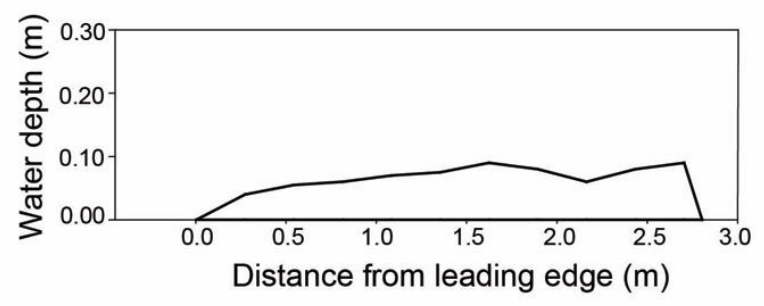

694 Figure 1 (A) Natural patches of Callitriche and (B) Groenlandia in the field. (C, D) Lateral view of the

695 two patches, with the black outline indicating canopy height at increasing distance from the patch

696 leading edge. 

A) Sparse-Dense staggered
$\bar{U}=0.15 \pm 0.03 \mathrm{~m} \mathrm{~s}^{-1}$
Turbulent KE $=0.016 \pm 0.008 \mathrm{~m}^{2} \mathrm{~s}^{-2}$

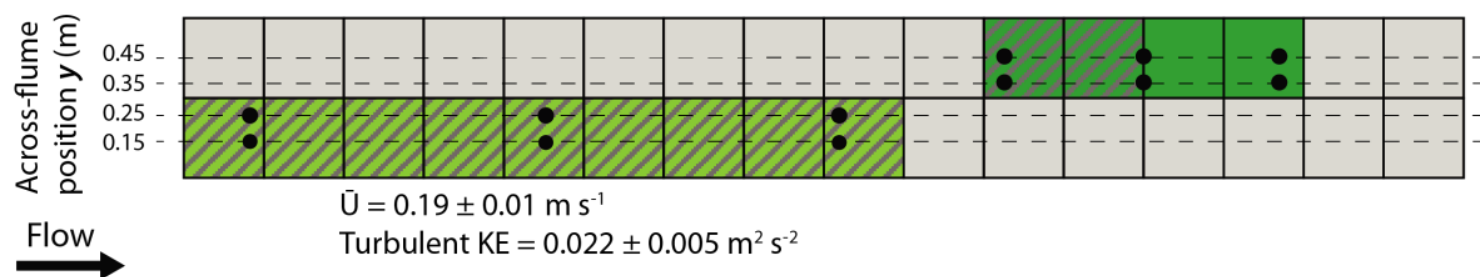

$\begin{array}{lll}\text { direction } & \text { B) Sparse-Dense aligned }\end{array}$

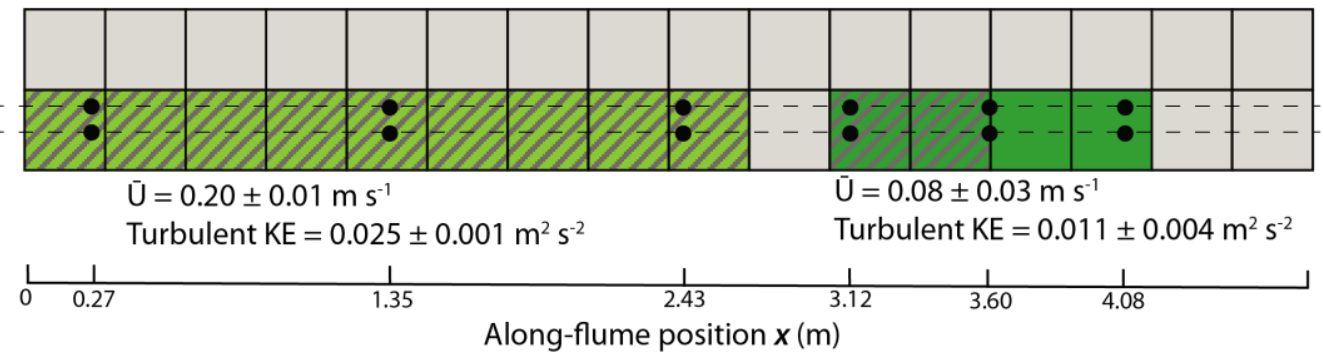

C) Dense-Sparse staggered

$\bar{U}=0.14 \pm 0.03 \mathrm{~m} \mathrm{~s}^{-1}$

Turbulent KE $=0.031 \pm 0.009 \mathrm{~m}^{2} \mathrm{~s}^{-2}$

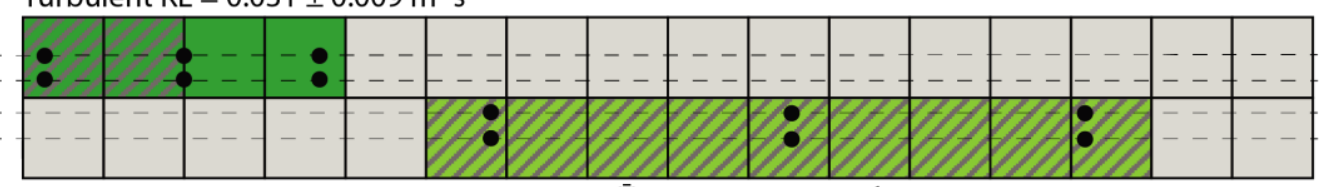

$\overline{\mathrm{U}}=0.20 \pm 0.01 \mathrm{~m} \mathrm{~s}^{-1}$

Turbulent KE $=0.034 \pm 0.011 \mathrm{~m}^{2} \mathrm{~s}^{-2}$

D) Dense-Sparse aligned

697

698 Figure 2 Schematic diagram of the four spatial configurations of aquatic macrophytes in the test

Along-flume position $\boldsymbol{x}(\mathrm{m})$

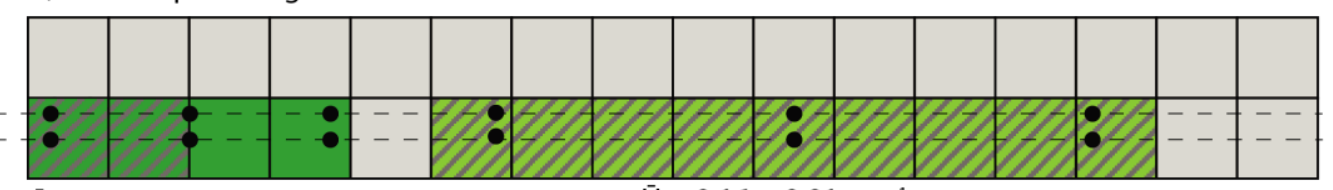

$\overline{\mathrm{U}}=0.17 \pm 0.02 \mathrm{~m} \mathrm{~s}^{-1}$

$\overline{\mathrm{U}}=0.16 \pm 0.01 \mathrm{~m} \mathrm{~s}^{-1}$

Turbulent KE $=0.033 \pm 0.014 \mathrm{~m}^{2} \mathrm{~s}^{-2}$

Turbulent KE $=0.042 \pm 0.010 \mathrm{~m}^{2} \mathrm{~s}^{-2}$

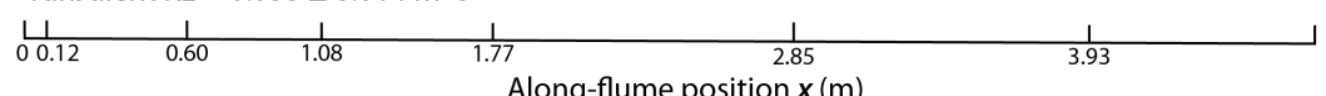

section of the flume. Light green indicates patches of Groenlandia (sparse canopy), and dark green

indicates patches of Callitriche (dense canopy). Diagonal lines indicate the boxes in which plants were

rooted. Black circles are locations of plant specimens removed after the incubations experiments for

assessment of $\mathrm{NH}_{4}^{+}$uptake rates, and of Acoustic Doppler Velocimeter (ADV) profile measurements.

Numbers indicate mean $( \pm \mathrm{SE})$ water velocity $\overline{\mathrm{U}}\left(\mathrm{m} \mathrm{s}^{-1}\right)$ and Turbulent Kinetic Energy (Turbulent KE) $\left(\mathrm{m}^{2} \mathrm{~s}^{-2}\right)$ within each species patch. 

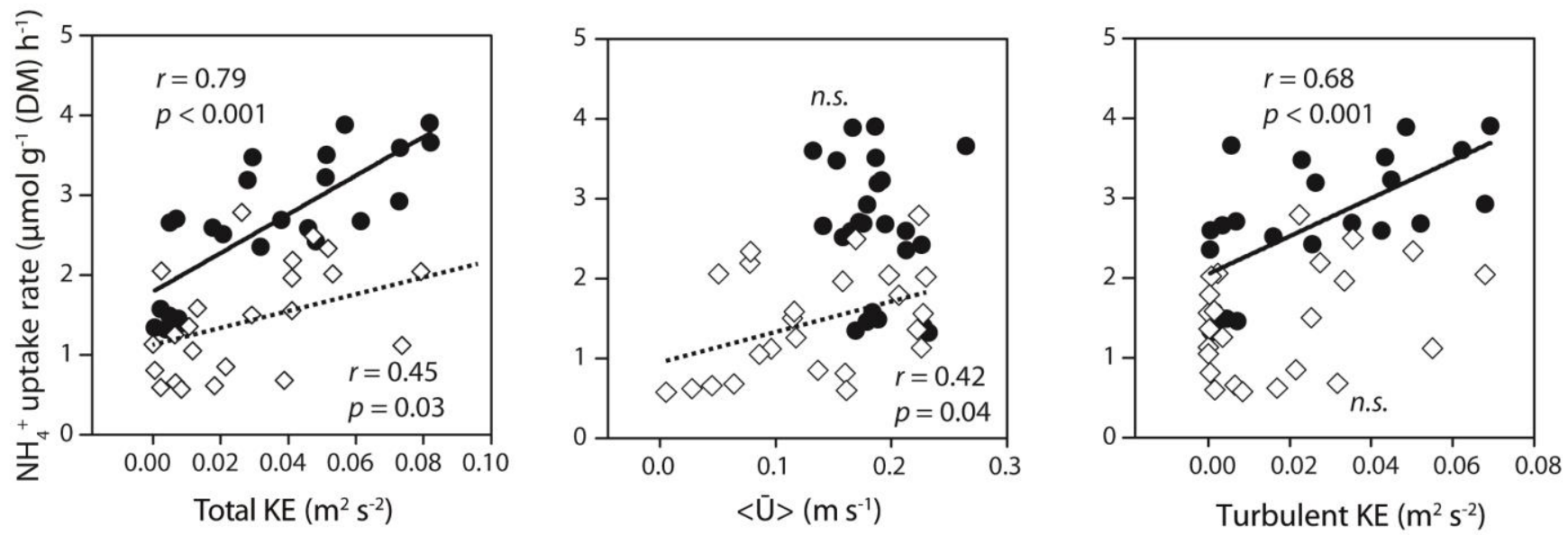

705

- Groenlandia (sparse) $\diamond$ Callitriche (dense)

706 Figure 3 Scatter plots of $\mathrm{NH}_{4}{ }^{+}$uptake rates $\left(\mu \mathrm{mol} \mathrm{g}{ }^{-1}(\mathrm{DM}) \mathrm{h}^{-1}\right)$ against Total Kinetic Energy (Total

$707 \mathrm{KE})\left(\mathrm{m}^{2} \mathrm{~s}^{-2}\right)$, depth-averaged velocity $\langle\overline{\mathrm{U}}\rangle\left(\mathrm{m} \mathrm{s}^{-1}\right)$ and Turbulent Kinetic Energy (Turbulent KE) $\left(\mathrm{m}^{2} \mathrm{~s}^{-}\right.$

$702^{2}$ ) for the sparse Groenlandia (black circles) and the dense Callitriche (white diamonds). Black lines

709 are linear regression lines for the Groenlandia (solid line) and Callitriche (dotted line) data separately

710 and represent significant relationships $(p \leq 0.05)$. 
(a) Callitriche

(dense species)

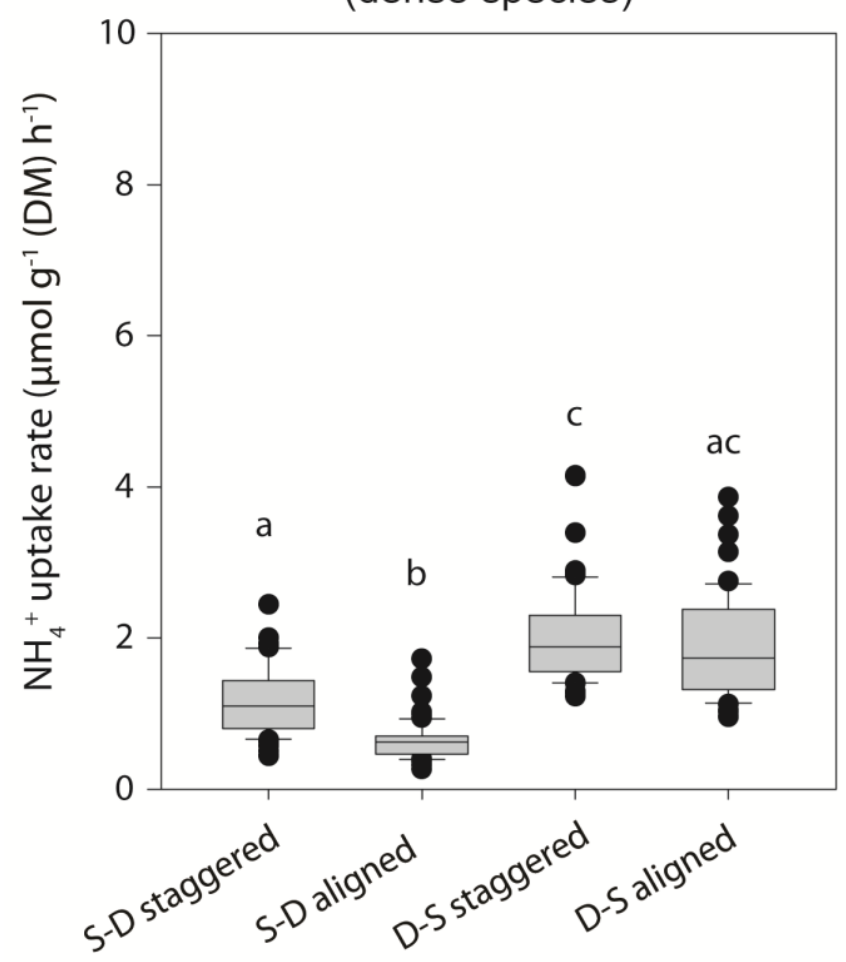

711

712

713

714

715

716

Configuration

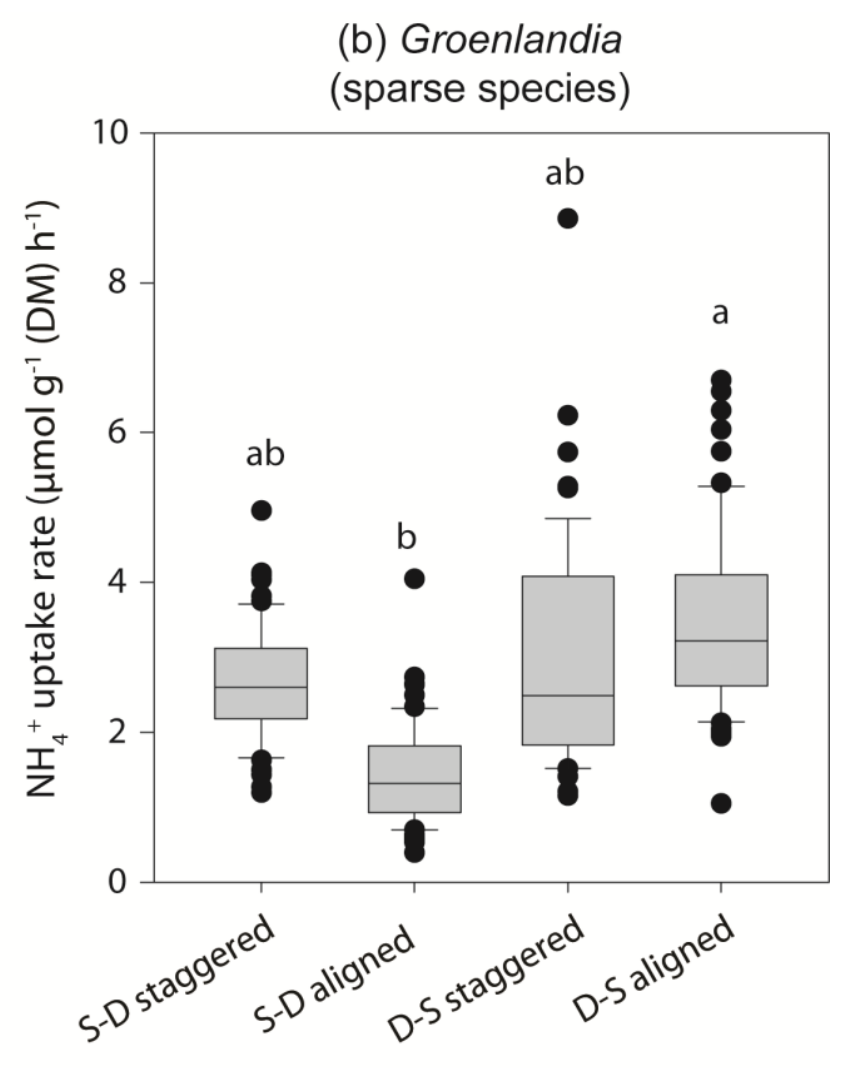

Configuration

(1) 


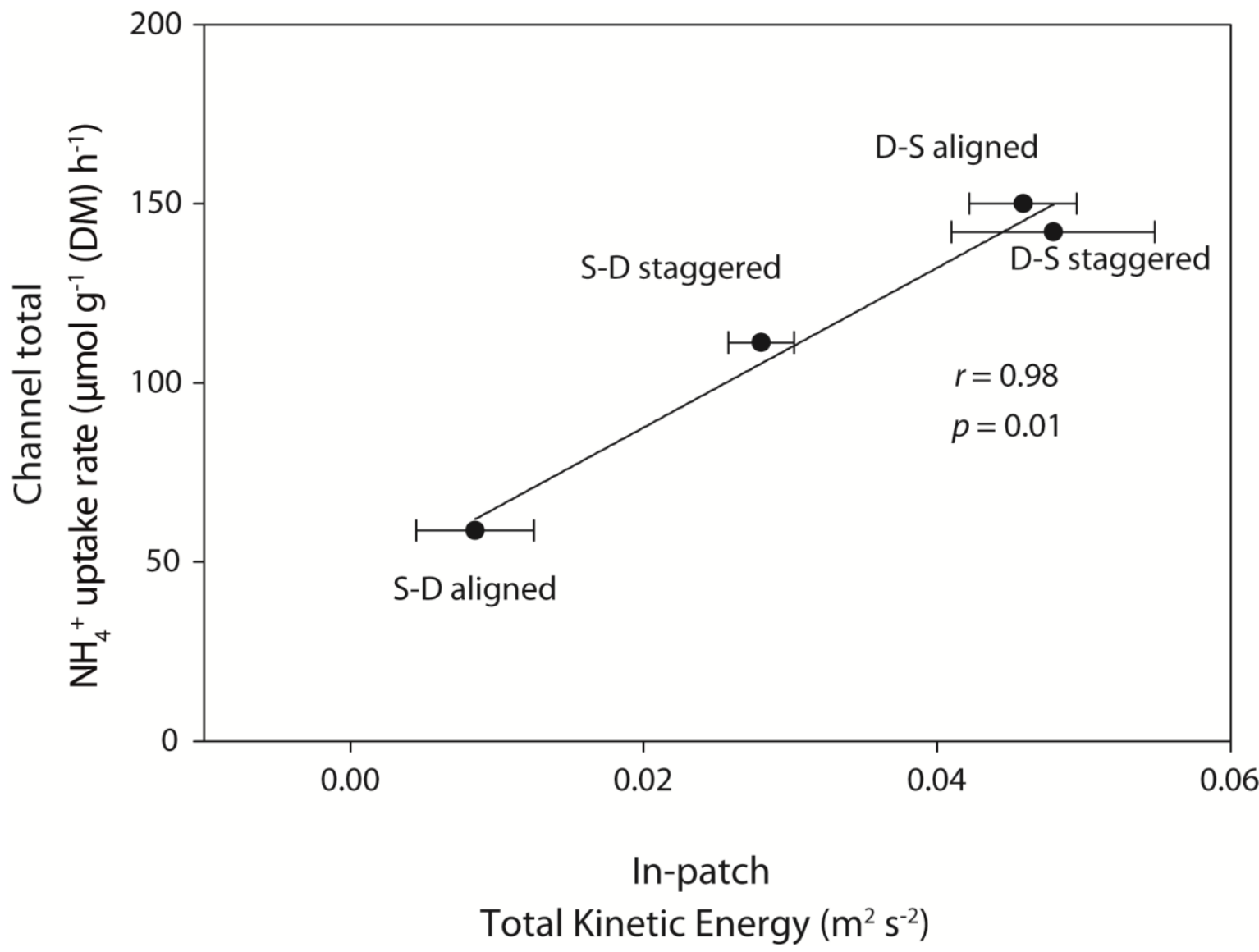

Figure 5 Scatter plots of channel total $\mathrm{NH}_{4}{ }^{+}$uptake rates $\left(\mu \mathrm{mol} \mathrm{g}^{-1}(\mathrm{DM}) \mathrm{h}^{-1}\right)$ in each spatial

719 configuration against Total Kinetic Energy $\left(\mathrm{m}^{2} \mathrm{~s}^{-2}\right)$ averaged within patches of Callitriche and

720 Groenlandia in each spatial configuration (S indicating sparse vegetation, D indicating dense

721 vegetation, see Figure 2). Error bars represent standard error of the mean. 


\section{(a) Sparse-Dense species configurations}

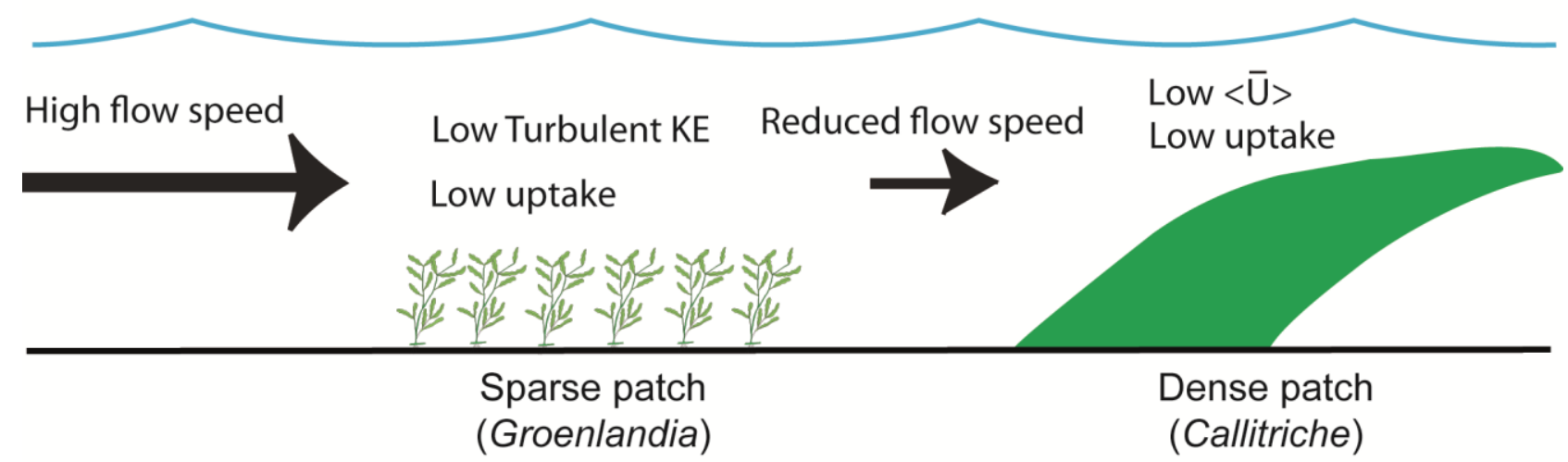

(b) Dense-Sparse species configurations

723

724

725

726

727

728

729

730

731

732

\section{High turbulence}

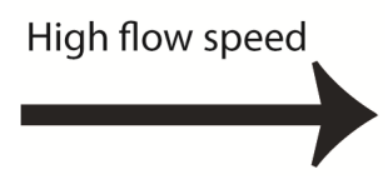

High $<\overline{\mathrm{U}}>$

High uptake

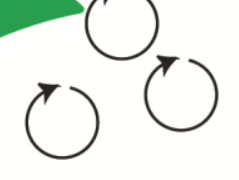

Dense patch

(Callitriche)
High Turbulent KE

High uptake

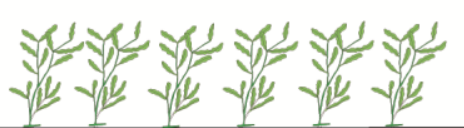

Sparse patch

(Groenlandia)

Figure 6 Schematized drawing of the effects of multispecific spatial patchiness on hydrodynamics and nutrient uptake rates. In Sparse-Dense configurations (a), the sparse vegetation is exposed to high mean flow but low turbulence, and does not benefit from being located at the leading edge. Similarly, the dense vegetation is exposed to low mean flow speed due to sheltering by the patch upstream, and hence has lower uptake rates. Instead, in Dense-Sparse configurations (b), uptake rates of both species are higher: the dense vegetation benefits from being at the leading edge and exposed to high mean flow speed (which increases uptake rates); at the same time, the sparse vegetation benefits from the high turbulence created in the wake of the dense patch. 


\section{Supporting Information}

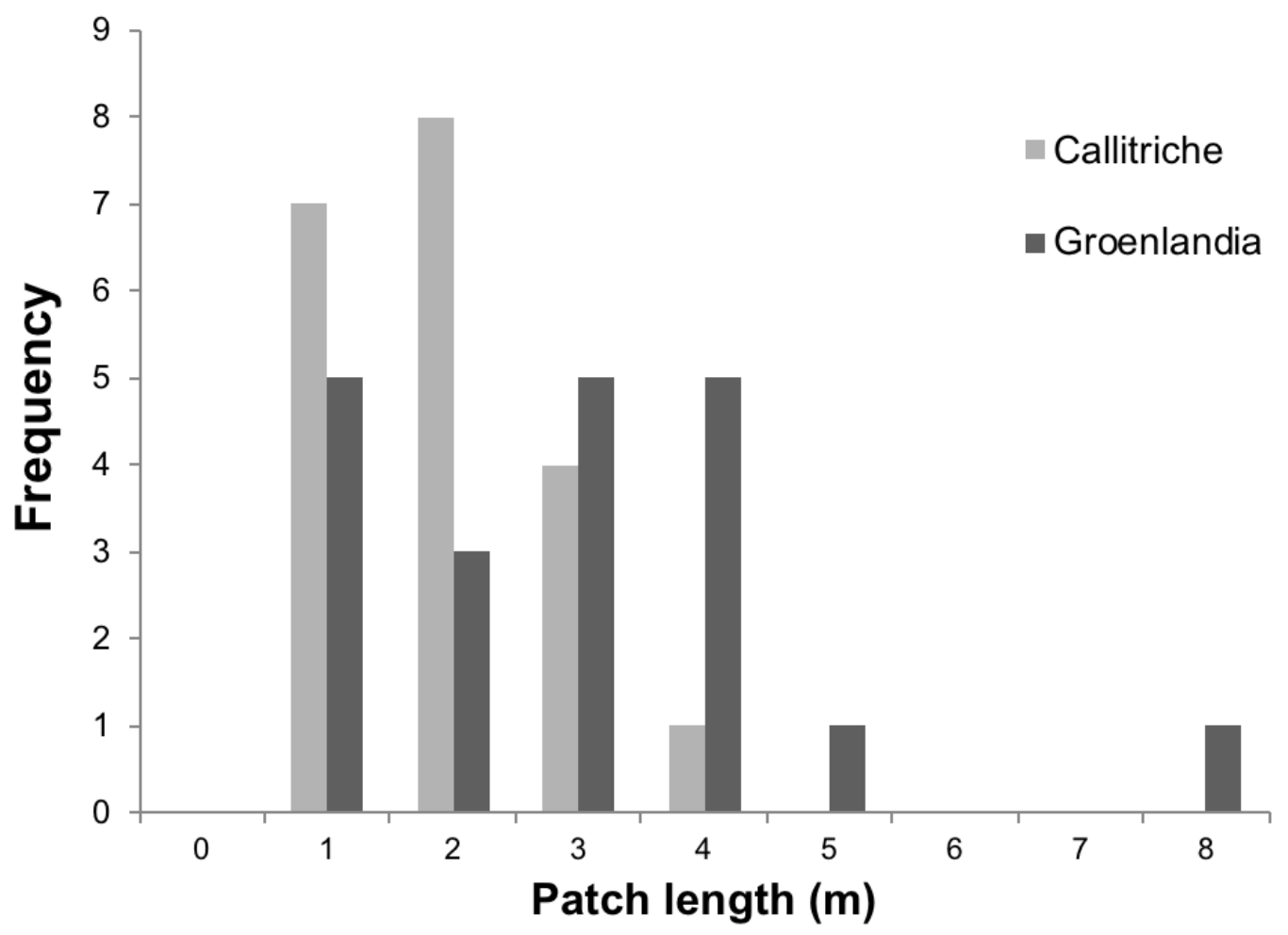

735 Figure S1: Frequency distribution of patch length $(\mathrm{m})$ for Callitriche and Groenlandia $(n=20)$ in field 736 conditions. 